\title{
Degradación y durabilidad de materiales rocosos de edificación en ambientes urbanos
}

\author{
Decay and durability of building stones in urban \\ environments
}

Fecha de recepción: 16-VI-98

Fecha de aceptación: 7-IX-98

\author{
C. M. GROSSI; R. M. ESBERT y F. DÍAZ-PACHE \\ Dpto. de Geología. Universidad de Oviedo
}

ESPAÑA

\section{RESUMEN}

Se estudia el efecto de ciertos agentes de contaminación presentes en ambientes urbanos $-\mathrm{SO}_{2}$ gaseoso, lluvia ácida y partículas sólidas- sobre rocas de naturaleza carbonatada y silicatada con características petrofisicas distintas, utilizadas en edificación.

Los ambientes seleccionados han sido Londres, Garston (UK) y Burgos, y los materiales expuestos han sido las calizas de Portland, Combe Down y Hontoria, la dolomia de Laspra y el granito Rosa Porriño. Se analizan los productos generados por la acción de los gases, la lluvia ácida y las partículas sólidas sobre los diferentes tipos de piedra. Se exponen los resultados obtenidos en la piedra expuesta a los ambientes antes citados, y los daños generados en algunos ensayos de envejecimiento artificial acelerado con el fin de resaltar las características intrínsecas de las piedras que condicionan su degradación.

Palabras clave: piedra de edificación, degradación, durabilidad, color, contaminación atmosférica, $\mathrm{SO}_{2}$, lluvia ácida, partículas sólidas, cristalización de sales, caliza, dolomía, granito.

\section{SUMMARY}

This paper studies the effect of some pollutant agents in urban environments -gaseous $\mathrm{SO}_{2}$, acid rain and solid particles- on carbonate and siliceous building stones with different petrophysic characteristics.

The selected environments have been London, Garston (UK) and Burgos (Spain) and the exposed materials have been Portland, Combe Down and Hontoria limestones, Laspra dolomite and Rosa Porriño granite. Products generated by the action of gases, acid rain and solid particles on stones have been analysed. To point out the properties of stones that influence their decay, the results obtained on the stones exposed to the above environments have been compared to those from some laboratory accelerated ageing tests.

Key words: building stone, stone decay, stone durability, stone colour, atmospheric pollution, $\mathrm{SO}_{2}$, acid rain, solid particles, salt crystallisation, limestone, dolomite, granite

\section{INTRODUCCIÓN}

La durabilidad podría definirse como la aptitud de un material rocoso para resistir la acción de los agentes de la intemperie (1). Depende de diversos factores que

\section{INTRODUCTION}

Durability is defined as the aptitude of a stone material to resist the action of outdoor agents (1). It depends on several factors that can be grouped in two main 
pueden englobarse en dos grandes categorías: intrínsecos y externos. Los intrínsecos dependen de los materiales y corresponden a las características petrográficas y físicas de los mismos (2). Los externos dependen fundamentalmente del ambiente. En éstos hay que tener en cuenta, además de su intensidad, la frecuencia de sus variaciones, es decir, su acción cíclica.

Entre los factores intrínsecos que condicionan la durabilidad de un material rocoso empleado para construcción, se encuentra la petrografía del material, es decir, su mineralogía, textura y composición química. La durabilidad también está condicionada por determinadas propiedades físicas, entre las que destaca la porosidad y configuración del sistema poroso, la superficie específica y las propiedades hídricas, relacionadas con el movimiento de fluidos en el seno del material (3). El sistema poroso condicionará el resto de las propiedades, por lo que su examen es esencial para una correcta interpretación de la durabilidad (4).

Entre los factores externos o ambientales, en áreas urbanas tienen especial relevancia los relacionados con los contaminantes gaseosos, líquidos o sólidos.

En este estudio se experimenta con rocas de edificación de distinta naturaleza y porosidad, expuest as a diferentes ambientes urbanos y sometidas a ensayos de laboratorio, con el fin de precisar sobre la incidencia de diferentes contaminantes: $\mathrm{SO}_{2}$ gas, lluvia ácida y partículas sólidas de contaminación.

\section{MATERIALES}

Los materiales utilizados han sido cuatro rocas cementadas, con espacios vacíos de tipo poro, y una roca cristalina, que presenta, preferentemente, espacios vacíos de tipo fisural. Concretamente se ha experimentado con rocas sedimentarias de naturaleza carbonatada, de distinta porosidad y configuración del sistema poroso: la dolomía de Laspra y las calizas de Hontoria, Portland y Combe-Down, y una roca cristalina de naturaleza silicatada y baja porosidad, el granito conocido comercialmente como Rosa Porriño.

Las cuatro primeras han sido utilizadas en España y Reino Unido como rocas monumentales. El granito, que procede de la zona de Porriño (Galicia), se emplea en España y es exportado a diversos países como roca ornamental.

En las tablas I y II se muestran los valores de la porosidad, distribución porométrica y superficie específica determinada mediante porosimetría de inyección de mercurio de las rocas citadas, así como categories: intrinsic (inherent) and external. The inherent ones depend on the materials and correspond to their petrographic and physical characteristics (2). The external ones mainly depend on the environment. In this case, it is necessary to take into account not only their intensity but also the frequency of their variations, that is, their cyclic action.

Among the inherent factors that influence durability of a building stone are the material petrography, that is, its mineralogy, texture and chemical composition and some physical properties. Porosity, pore size distribution, specific surface area and properties related to moisture transfer are pointed out among these physical properties (3). Pore network will determine the remaining the properties. Therefore, its analysis is essential for a correct interpretation of the durability (4).

Among the external or environmental factors, those related to gaseous, liquid or solid pollutants are of special relevance in urban areas.

In order to specify the incidence of the above mentioned pollutants (gaseous $\mathrm{SO}_{2}$, acid rain and solid particles from atmospheric pollution) some building stones of different nature and porosity have been exposed to diverse urban environments and laboratory tests.

\section{MATERIALS}

The studied materials have been four cemented rocks with voids of the type "pore" and a crystalline rock with voids mainly of the type "fissure". To be precise the cemented stones have been carbonate sedimentary rocks of different porosity and pore size distribution: Laspra dolomite and Hontoria, Portland and CombeDown limestones. The crystalline rock is a siliceous stone of low porosity: the granite known in the market as Rosa Porriño.

The first four stones have been used in Spain and in the UK as monumental stones. The granite is used in Spain and exported to different countries as an ornamental stone.

Tables I and II show the values of porosity, pore size distribution and specific surface area or the stones determined by mercury porosimetry and several physical properties related to moisture transfer which 
TABLA I (TABLE /)

Porosidad (porosimetría de mercurio)/Porosity (mercury porosimetry)

\begin{tabular}{|l|c|c|c|}
\hline & $\begin{array}{c}\text { Porosidad abierta } \\
\text { (Open porosity) } \\
n_{0}(\%)\end{array}$ & $\begin{array}{c}\text { Poros con radio } \\
\text { (Pore size) } \\
<1 \mu \mathrm{m}(\%)\end{array}$ & $\begin{array}{c}\text { Superficie especifica } \\
\text { (Specific surface area) } \\
\left(\mathrm{m}^{2} / \mathrm{g}\right)\end{array}$ \\
\hline LASPRA & $\approx 31$ & 94,4 & 4,03 \\
\hline HONTORIA & $\approx 20$ & 29,5 & 0,26 \\
\hline PORTLAND & $\approx 20$ & 51,6 & 1,21 \\
\hline COMBE DOWN & $\approx 28$ & 70,7 & 1,38 \\
\hline ROSA PORRINO & $\approx 0,8$ & 4,5 & 0,54 \\
\hline
\end{tabular}

Grossi et al. 1995 (30)

TABLA \| (TABLE $\|$ )

Propiedades físicas (Physical properties)

\begin{tabular}{|l|c|c|c|c|c|}
\hline & $\begin{array}{c}\text { Absorción libre de } \\
\text { agua) } \\
\text { (Free water } \\
\text { absorption) } \\
(\%)\end{array}$ & $\begin{array}{c}\text { Capilaridad } \\
\text { (Capillarity) } \\
\left(\mathrm{kg} \cdot \mathrm{m}^{2} \cdot \mathrm{min}^{1 / 2}\right) \times 10^{-2}\end{array}$ & $\begin{array}{c}\text { Permeabilidad al } \\
\text { vapor de agua } \\
\text { (Water vapour } \\
\text { permeability) } \\
\left(\mathrm{g} \cdot \mathrm{m}^{-1} \cdot \mathrm{h}^{-1} \cdot \mathrm{mmHg}^{-1}\right)\end{array}$ & $\begin{array}{c}\text { Captación de } \\
\text { vapor de agua } \\
\text { (Hydroscopic } \\
\text { mixture content) } \\
\text { HR }=60 \%\end{array}$ \\
\hline LASPRA & 12 & $56-45$ & $1,3 \times 10^{-3}$ & 12,5 & 113,3 \\
\hline HONTORIA & 6 & 80 & $1,0 \times 10^{-3}$ & 0,05 & 0,24 \\
\hline PORTLAND & 7,5 & 70 & $0,90 \times 10^{-3}$ & 0,38 & 2,8 \\
\hline COMBE DOWN & 9 & 28 & $0,9 \times 10^{-3}$ & 0,81 & 3,6 \\
\hline ROSA PORRIÑO & 0,37 & 0,02 & $0,25 \times 10^{-3}$ & $-\cdots$ & $\cdots$ \\
\hline
\end{tabular}

Grossi et al. 1995 (30)

una serie de propiedades hídricas que pueden condicionar la respuesta del material al medio ambiente: absorción libre de agua (Normal 7/81); permeabilidad al vapor de agua (Normal 21/85); captación de vapor de agua o higroscopicidad y capilaridad (Normal 11/85) (Tabla II).

Atendiendo a su porosidad los materiales carbonatados seleccionados se podrían dividir en dos grupos: uno, de muy alta porosidad (Laspra y Combe-Down, $\mathrm{n}_{0} \approx 30 \%$ ); $\mathrm{y}$ otro de porosidad alta (Hontoria y Portland, $\mathrm{n}_{0}=20 \%$ ). El granito corresponde a un material cristalino de porosidad baja (Rosa Porriño $n_{0}=0,8 \%$ ).

De los valores de las tablas I y II se desprende que los valores de la absorción de agua están en relación con los de la porosidad abierta. El resto de las propiedades, can influence the stone response to the environment: free water absorption (Normal 7/81); water vapour permeability (Normal, 2/85); hygroscopic moisture content and capillary suction (Normal 11/85) (Table II).

The carbonate stones could be divided in two groups according to their porosity: one of very high porosity (Laspra and Combe Down; $n_{o}=30 \%$ ) and another of high porosity (Hontoria and Portland, $n_{0}=20 \%$ ). Rosa Porriño granite is a crystalline material of low porosity $\left(n_{0}=0,8 \%\right)$

From the values of tables I and II it can be stated that water absorption values are related to those of open porosity. Though, depending on porosity value, the 
aunque dependen del valor de la porosidad, están relacionadas, principalmente, con la distribución porométrica. Especialmente indicativa es la captación de vapor de agua que está claramente determinada por los valores de la superficie específica. Se puede observar cómo es mucho más elevada en Laspra, que es la roca con mayor superficie específica. En esta roca parte de esta humedad es debida a las sales higroscópicas que contiene (5).

\section{DEGRADACIÓN DE MATERIALES ROCOSOS EN AMBIENTES URBANOS}

El proceso natural de degradación de las piedras de edificación se ve acelerado, en zonas industriales y urbanas, por la elevación de los niveles de contaminación (6-12).

La acción de los contaminantes puede relacionarse con el depósito de gases ácidos y de materia particulada en la superficie de la piedra. La principal contribución de los primeros será la reacción con el material, hecho muy destacado en las rocas carbonatadas, mientras que la materia particulada contribuirá al ennegrecimiento de la piedra y podrá favorecer la oxidación del $\mathrm{SO}_{2}$ a sulfato y el ulterior ataque químico de éste a la piedra.

La deposición seca del $\mathrm{SO}_{2}$ gaseoso, procedente fundamentalmente de la combustión de combustibles fósiles, y su subsecuente oxidación a sulfato es un agente de deterioro muy importante en zonas urbanas. Otros gases presentes en ciudades, como el $\mathrm{NO}_{x}$, pueden también generar depósitos en la superficie de la roca (13). En presencia de humedad el $\mathrm{NO}_{\mathrm{x}}$ puede favorecer la oxidación del $\mathrm{SO}_{2}(14)$.

El nivel de absorción del $\mathrm{SO}_{2}$ por el material, dependerá de la humedad relativa (14-18) y, por tanto, del contenido en humedad higroscópica de la roca que será mayor cuanto mencr sea el tamaño de poro y mayor la superficie específica (4). La presencia de sales higroscópicas en materiales porosos contribuye a mantener la capa de humedad en el interior de los mismos, facilitando por tanto la absorción y oxidación $\operatorname{del~SO}(19,20$ y 21$)$.

La degradación de los materiales pétreos por la materia particulada (partículas sólidas) es debida, entre otros y fundamentalmente a dos mecanismos: a su efecto catalizador en la oxidación del $\mathrm{SO}_{2}$ a sulfato, en presencia de humedad, y al ennegrecimiento ("soiling") que su depósito genera en la piedra. Este ennegrecimiento es debido principalmente al depósito de materia carbonosa de combustión, que, actualmente, en zonas urbanas, tiene su fuente principal en los motores diesel (22 y 23). La medida del remaining properties are mainly related to porometric distribution. Specially significant is the taking of water vapour which is clearly determined by the values of the specific surface. It can be seen that is much higher in Laspra, that is the rock with higher specific surface. Some of the moisture in this rock is due to the hygroscopic salts it contains (5).

\section{DECAY OF BUILDING STONES IN URBAN ENVIRONMENTS}

The decay of building stones is a natural process that is accelerated on urban or industrial areas due to the high levels of atmospheric pollution (6-12).

The action of pollutants can be related to the deposit of acidic gases and particulate matter on the stone surfaces. Gases react with stones, especially in the case of carbonate stones. Particulate matter contributes to the soiling of the stone and can accelerate the oxidation of $\mathrm{SO}_{2}$ to $\mathrm{SO}_{4}=$

Dry deposition of $\mathrm{SO}_{2}$, mainly coming from the combustion of fossil fuels, and the subsequent oxidation into sulphate is a very important decay agent in urban areas. Other gases present in urban atmospheres, such as $\mathrm{NO}_{x}$, can also deposit on stone surfaces (13). In presence of moisture, $N O_{x}$ can favour $\mathrm{SO}_{2}$ oxidation (14).

The rate of uptake of $\mathrm{SO}_{2}$ by the material, depends on the relative humidity (14-18) and, therefore, on the hygroscopic moisture content of the stone which will be higher at smaller pore size and higher specific surface area (4). The presence of hygroscopic salts inside porous materials contributes to keep the moisture layer inside them, making $\mathrm{SO}_{2}$ uptake and oxidation easier $(19,20,21)$.

Stone decay by particulate matter (solid particles) is mainly due to two mechanisms: its catalytic effect in the oxidation of $\mathrm{SO}_{2}$ to sulphate (in the presence of moisture) and the soiling of stone surfaces. This black soiling is mostly due to the deposition of carbonaceous matter from combustion. Nowadays diesel engines are the main source of carbon $(22,23)$. Changes in surface reflectance are commonly used to measure soling. Porosity and surface roughness are 
ennegrecimiento generalmente se determina por la variación de reflectancia de la superficie. Entre las características de la superficie del material rocoso que favorecen el depósito de partículas se pueden citar sus espacios vacíos (porosidad) y la rugosidad superficial.

Los productos de reacción entre la piedra y la contaminación atmosférica también pueden deteriorar el material. Son fundamentalmente sales solubles, entre las que destacan los sulfatos, aunque también pueden citarse cloruros y nitratos. Las sales pueden contribuir al deterioro por ciclos de cristalizacióndisolución; hidratación-deshidratación; por expansión térmica diferencial en relación con el material del sustrato que las contiene; o por cambios texturales o estructurales de la misma sal (24 y 25).

\subsection{Metodología experimental: exposición del material a diferentes ambientes}

Para estudiar la degradación de los materiales de edificación seleccionados se han realizado ensayos de exposición in situ en diferentes ambientes urbanos. Los resultados de este trabajo corresponden, principalmente, a los datos obtenidos en Londres, Garston (U.K.) y Burgos.

Para el estudio de las transformaciones experimentadas en las piedras -material alterado- se han realizado análisis con microscopía electrónica de barrido (SEM) y microanálisis (EDX); espectroscopía de infrarrojos con transformada de Fourier; difracción de rayos $\mathrm{X}$; medidas de colorimetría y brillometría. La cromatografia iónica se ha utilizado para el análisis de aniones y cationes. También se han medido las variaciones de peso entre las piedras sin alterar y las alteradas.

Para la medida de la concentración de $\mathrm{SO}_{2}$ en el aire, se han seguido diferentes métodos, entre ellos el uso de una sonda que mide, por absorción de radiación ultravioleta, la concentración de $\mathrm{SO}_{2}$. Las partículas sólidas se han recogido periódicamente en Burgos con un captador de impacto en cascada, así como sobre soportes de piedra que fueron colocados tanto expuestos como protegidos del agua de lluvia.

\subsubsection{Acción de los gases y lluvia ácida:} exposición de la piedra en Londres y Garston

El objetivo fue determinar la respuesta de los cuatro materiales carbonatados porosos a los gases ácidos ( $\mathrm{SO}_{2}$ fundamentalmente). Para ello, se han expuesto durante un año probetas de $5 \times 5 \times 1 \mathrm{~cm}$ en dos ambientes: uno urbano, centro de Londres (en el exterior de la Abadía de Westminster) y otro some of the material characteristics that determine particulate deposition.

Products from reaction between stone and atmospheric pollution can also deteriorate materials. These products are mainly soluble salts, being sulphates some of the most characteristic, although chlorures and nitrates can be also found. Salts can contribute to the decay due to cycles of crystallisation-dissolution; cycles of hydration-dehydration, differential thermal expansion to the host material or salt textural or structural changes $(24,25)$.

\subsection{Experimental methodology: material exposure to different environments}

To study building material degradation, some on-site exposure tests in different urban environments have been carried out. The results presented in this paper mostly correspond to data obtained in London, Garston and Burgos.

To study changes in stones -decay material-some analyses have been undertaken by scanning electron microscopy (SEM) and microanalysis (EDX), FourierTransform infrared spectroscopy and X-Ray difractometry and measurements with colourimetry and glossmetry. Ionic chromatography has been used to analyse anions and cations. The weight variation of decayed stones in relation to non-decay stones has been determined as well.

To measure the atmospheric concentration of $\mathrm{SO}_{2}$, some methods have been used; among them a probe which measures $\mathrm{SO}_{2}$ concentration by ultraviolet radiation absorption. Solid particles have been both collected by a cascade impactor sampler and deposited on stones samples placed in horizontal positions both exposed to and sheltered from rainfall.

\subsubsection{Action of gases and acid rain: stone exposure in London and Garston}

The aim was to assess the response of the four porous carbonate stones to acidic gases (mainly $\mathrm{SO}_{2}$ ). Specimens $(5 \times 5 \times 1 \mathrm{~cm})$ were subjected to one year exposure to two enviroments: urban, central London (exterior of Westminster Abbey) and suburban 
suburbano, Garston-Hertfordshire (plataforma de exposición en el Building Research Establishment).

Se ha seguido la metodología usada por investigadores del Building Research Establishment (BRE) (26). La mitad de las muestras se colocaron protegidas de la lluvia y, por lo tanto, sometidas solamente a deposición seca. El resto fue colocado en lugares no protegidos de la lluvia y expuestos, por tanto, a deposición tanto seca como húmeda.

La valoración se ha realizado estudiando el material neoformado, en las muestras protegidas de la lluvia, y calculando la pérdida de material en superficie (recesión superficial) de las muestras expuestas a la lluvia.

En muestras protegidas de la lluvia se ha detectado, mediante análisis por cromatografía iónica, una gran cantidad de sulfatos, que corresponden a sulfatos de calcio (Figura 1). En mucha menor proporción se han detectado cloruros, nitratos y oxalatos. Los sulfatos de neoformación tienden a concentrarse en las capas más superficiales del material, mientras que los nitratos y cloruros, generalmente más solubles e higroscópicos, tienden a distribuirse más uniformemente por el interior de las muestras (Figura 2) (27-29). En las muestras sometidas a la acción de la lluvia, la concentración de sulfatos es mucho menor debido a posteriores procesos de disolución.

La recesión superficial en muestras expuestas a la lluvia (Tabla III) es debida a pérdida de carbonato por deposición seca de $\mathrm{SO}_{2}$, por lluvia ácida y por disolución natural del material (acción de lluvia no contaminada). El porcentaje de recesión superficial
(Garston-Hertforshire: exposure site at Building Research Establishment -BRE-).

At each of these sites, pairs of freely rotating carousels of stone tablets $(5 \mathrm{~cm} \times 5 \mathrm{~cm} \times 1 \mathrm{~cm})$ were installed following the methodology used by researchers at the $B R E$ (26). One of the carousels was sheltered from rainfall and exposed only to dry deposition. The other was exposed to wet and dry deposition.

To evaluate stone decay, new formed material in sheltered stones has been analysed and its weight loss (surface recession) has been determined in unsheltered stone samples.

A great amount of sulphates that correspond to calcium sulphates (Figure 1) were detected, by means of ionic chromatography, in sheltered samples. Nitrates, chlorides and oxalates were also detected but in much lower proportion. Newly formed sulphates tend to concentrate in the first layers of the stone, whereas nitrates and chlorides which generally are more soluble and hygroscopic tend to evenly distribute across the sample (Figure 2) (27-29). In the specimens subjected to rain action the sulphate concentration is less due to dissolution processes.

Surface recession in unsheltered samples (Table III) is due to loss of carbonate by $\mathrm{SO}_{2}$ dry deposition, acid rain and natural dissolution (non polluted rain). The relative percentage of carbonate loss due to dry deposition, acid rain and natural dissolution (Table IV)

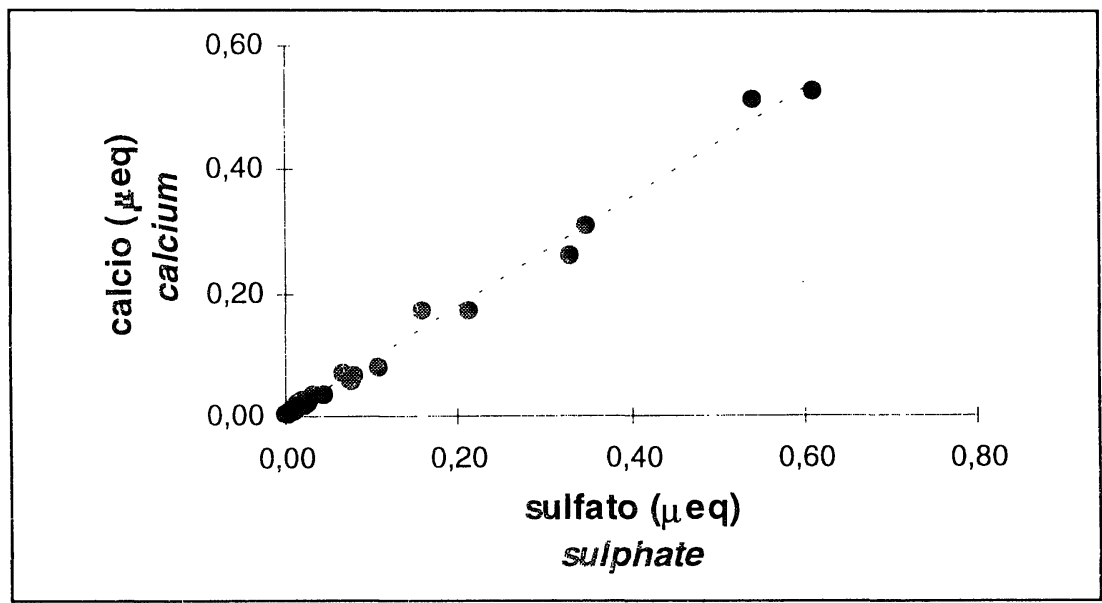

Figura 1.- Relación entre los sulfatos y el calcio analizados por cromatografia iónica en rocas carbonatadas (expuestas al ambiente).

Figure 1.- Relation between sulphates and calcium analysed by ion chromatography in carbonate stones (outdoors exposure). 

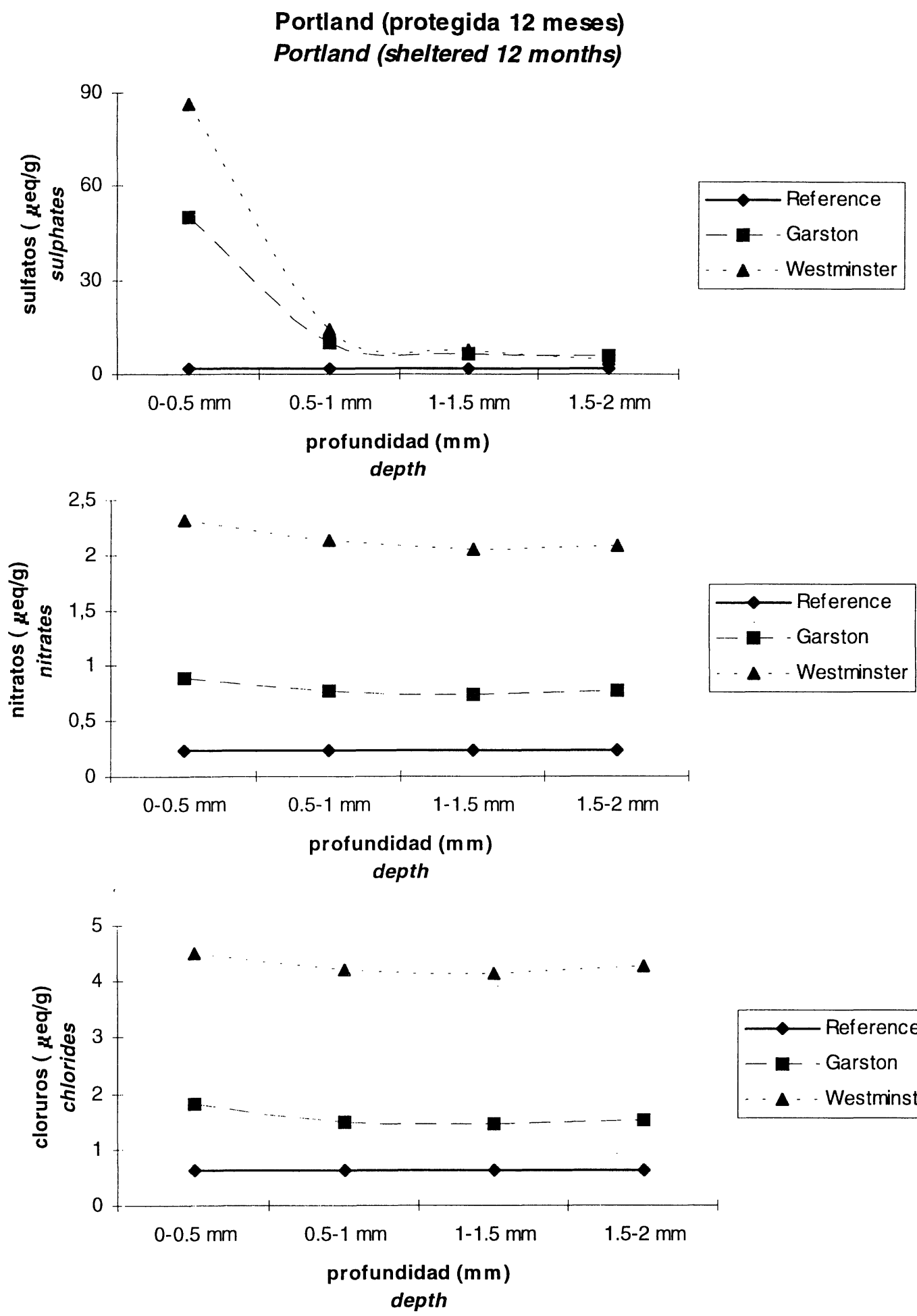

Figura 2.- distribución de sulfatos, nitratos y cloruros en el interior de la caliza de Portland (un año de exposición in situ).

Figure 2.- Distribution of sulphates, nitrates and chlorides inside Portland limestone (a year on-site exposure). 
TABLA III (TABLE III)

Recesión superficial ( $\mu \mathrm{m} / \mathrm{año})$ (Surface recession rates ( $\mu \mathrm{m} / \mathrm{year})$ )

\begin{tabular}{||l|c|c||}
\hline & GARSTON & LONDRES (LONDON) \\
\hline LASPRA & 41,1 & 47,6 \\
\hline HONTORIA & 11,8 & 11,4 \\
\hline PORTLAND & 12,0 & 13,9 \\
\hline COMBE DOWN & 40,8 & 42,7 \\
\hline
\end{tabular}

Grossi et al. 1995 (30)

TABLA IV (TABLE IV)

Principales causas de pérdida de carbonato (Processes leading carbonate loss)

\begin{tabular}{|c|c|c|c|c|c|c|c|c|}
\hline \multirow[t]{2}{*}{$\begin{array}{l}\text { Tipo de piedra } \\
\text { (Type of stone) }\end{array}$} & \multicolumn{2}{|c|}{$\begin{array}{l}\text { Carbonato perdido } \\
\text { por deposición } \\
\text { (Loss of carbonate } \\
\text { by dry deposition) } \\
\text { (g) }\end{array}$} & \multicolumn{2}{|c|}{$\begin{array}{l}\text { Deposición seca } \\
\text { (Dry deposition) } \\
\text { (\%) }\end{array}$} & \multicolumn{2}{|c|}{$\begin{array}{l}\text { Lluvia ácida } \\
\text { (Acid rain) } \\
\text { (\%) }\end{array}$} & \multicolumn{2}{|c|}{$\begin{array}{c}\text { Disolución natural } \\
\text { (Natural disolution) } \\
\text { (\%) }\end{array}$} \\
\hline & $\begin{array}{l}\text { Londres } \\
\text { ILondon }\end{array}$ & $\begin{array}{l}\text { Garston } \\
\text { GarstonI }\end{array}$ & $\begin{array}{l}\text { Londres } \\
\text { ILondon }\end{array}$ & $\begin{array}{l}\text { Garston } \\
\text { GarstonI }\end{array}$ & $\begin{array}{l}\text { Londres } \\
\text { ILondon }\end{array}$ & $\begin{array}{l}\text { Garston } \\
\text { GarstonI }\end{array}$ & $\begin{array}{l}\text { Londres } \\
\text { ILondon }\end{array}$ & $\begin{array}{l}\text { Garston } \\
\text { Garston/ }\end{array}$ \\
\hline LASPRA & 0,13 & 0,04 & 19,0 & 8,4 & 3,3 & 3,8 & 77,7 & 87,8 \\
\hline HONTORIA & 0,03 & 0,02 & 20,7 & 11,3 & 6,5 & 6,3 & 72,8 & 82,4 \\
\hline PORTLAND & 0,04 & 0,02 & 20,7 & 11,8 & 5,3 & 6,2 & 74,0 & 82,0 \\
\hline COMBE DOWN & 0,07 & 0,04 & 10,7 & 6,6 & 1,9 & 2,0 & 87,4 & 91,4 \\
\hline
\end{tabular}

Grossi et al. 1995 (30)

debida a la deposición seca de $\mathrm{SO}_{2}$ se ha calculado a partir de la determinación de la cantidad de yeso (formado a partir de la deposición de $\mathrm{SO}_{2}$ ).

Relacionando esta recesión con la cantidad de yeso determinado en muestras protegidas de la lluvia, combirtiéndolo en peso equivalente de calcita o dolomita y siguiendo algunos métodos como el de Webb et al. (1992) (21) se ha estimado el porcentaje relativo de pérdida de carbonato debido a deposición seca, lluvia ácida y disolución natural del material (Tabla IV). Se ha asumido que la deposición seca es igual en muestras expuestas y protegidas, aunque en el caso de las expuestas, el yeso formado será lavado posteriormente por el agua de lluvia.

Se observa que hay una fuerte relación entre la recesión superficial y la porosidad abierta (Tablas I y III). Por otra parte, en la tabla IV se observa como la disolución was estimated by relating surface recession to the amount of gypsum determined in sheltered samples and following some methods such as Webb et al. (1992) (21) it is assumed that dry deposition is equivalent in sheltered and non sheltered specimens, although in the exposed ones the gypsum is washed by rainwater (Table IV).

There is a relation between surface recession and open porosity (Tables I and III). Dissolution by unpolluted water is the most important process (30) 
debida al agua no contaminada es el proceso más importante (30). Los poros abiertos de las rocas favorecen la penetración de agua y, por tanto, a medida que su número aumenta, se puede disolver más carbonato de forma natural. La mayor parte de la pérdida de material, hasta casi el $90 \%$ en algunos casos, es debida a la disolución por agua de lluvia no contaminada (disolución natural del carbonato), mientras que sólo un pequeño porcentaje (3-8\%) es debido a la disolución del carbonato por lluvia ácida. El resto (10-20\%) es debida a la disolución del yeso formado por deposición seca de $\mathrm{SO}_{2}$ y reacción con el carbonato cálcico de la roca. Por tanto, la deposición seca de $\mathrm{SO}_{2}$ contribuye más que la deposición humeda al ataque químico de caliza por deposición ácida (20).

El porcentaje relativo de pérdida por deposición seca dependerá de la concentración de $\mathrm{SO}_{2}$ en la atmósfera. La lluvia ácida tiene una menor repercusión en la pérdida de material en estas rocas (20).

\subsubsection{Depósito de partículas sólidas de} contaminación: exposición de la piedra en Burgos

Los ensayos se han realizado principalmente para analizar el depósito de partículas sólidas de contaminación y sus efectos sobre las piedras de edificación. El estudio se ha llevado a cabo en la Fachada de Coronería (orientación OSO) de la catedral de Burgos, edificio elegido como monumento piloto. Se han expuesto muestras de roca de dolomía de Laspra, caliza de Hontoria y granito Rosa Porriño. Las probetas $(5 \times 5 \times 1 \mathrm{~cm})$ se han colocado en posición horizontal, tanto expuestas como protegidas de la lluvia.

Como se citó anteriormente la degradación de los materiales pétreos por el depósito de partículas sólidas se traduce fundamentalmente en: a) su efecto catalizador en la oxidación del $\mathrm{SO}_{2}$ a sulfato, en presencia de humedad (23), y b) en el ennegrecimiento ("soiling") de la piedra.

Las características de las partículas de un ambiente determinado, especialmente su composición química, influirán, de manera significativa, en su acción como agentes de deterioro de la piedra. Para profundizar en el conocimiento de las partículas existentes en el entorno de la Catedral de Burgos (31) se realizaron varios muestreos de partículas atmosféricas del aire empleando un captador de impacto en cascada. Este muestreardor las separa en tres grupos atendiendo a sus tamaños: de 0,05 a $0,3 \mu \mathrm{m}$; de 0,3 a $2 \mu \mathrm{m}$; y mayores de $2 \mu \mathrm{m}$ (hasta un máximo de $35 \mu \mathrm{m}$ ).
(Table IV). Stone open pores favour water penetration and then, the higher the porosity is, the more carbonate can be dissolved in a natural way. Most weight loss (until 90\%) is due to natural dissolution, whereas only a small percentage (3-8\%) is due to acid rain. The remaining (10-20\%) is due to the dissolution of the gypsum formed by the reaction between the dry deposition of $\mathrm{SO}_{2}$ and the stone calcium carbonate. Consequently, the dry deposition of $\mathrm{SO}_{2}$ contributes more than wet deposition to the chemical attack of limestones by acid deposition (20).

The relative percentage of weight loss due to dry deposition will depend on the atmospheric concentration of $\mathrm{SO}_{2}$. In these stones the acid rain has a minor influence on the material loss (20).

\subsubsection{Deposition of solid particles from atmospheric pollution: stone exposure at Burgos}

Tests have been carried out mainly to analyse the deposition and effect of solid particles from atmospheric pollution on building stones. The study has been undertaken at Coronería Façade (Orientation WSW) of Burgos cathedral which has been selected as example monument. Samples $(5 \times 5 \times 1 \mathrm{~cm}$ and $1 x 1 x 1 \mathrm{~cm}$ ) of Laspra dolomite, Hontoria limestone and Rosa Porriño granite have been placed in horizontal positions both sheltered and exposed to rainfall.

As mentioned above, the effects of particle deposition on stone degradation are their catalytic effect of particles in the oxidation of $\mathrm{SO}_{2}$ into sulphate (23) and the soiling of the stone.

Particle characteristics of a specific environment, especially their chemical composition, will significantly influence their decay effect on stone. To go deeply into the knowledge of the particles around Burgos cathedral (32) several samples of atmospheric particles have been taken by a "captador de impacto en cascada". The collector separates particles in three groups according to their size: from 0.05 to $0.3 \mu \mathrm{m}$; from 0.3 to $2 \mu \mathrm{m}$; and bigger than $2 \mu \mathrm{m}$ (up to amaximum of $35 \mu \mathrm{m}$ ). 
Con los datos obtenidos de ambos estudios -exposición y captación- se puede conocer la naturaleza de las partículas y su participación en el deterioro de las piedras de edificación.

Las partículas muestreadas con el captador han sido analizadas empleando SEM + EDX, así como un programa que permite la caracterización automática de las mismas -Featurescan- (Figura 3). Los resultados más significativos se recogen en la tabla $\mathrm{V}$, en la que se clasifican las partículas atendiendo a su tamaño y composición química.

Los resultados indican que:

- Las partículas pequeñas (entre 0,05 y $0,3 \mu \mathrm{m}$ ) aparecen formando agregados donde el azufre es el elemento mayoritario (en ocasiones casi exclusivo), acompañado por pequeñas cantidades de calcio $y / 0$ potasio (Figura 4). Estas partículas de sulfato, procedentes probablemente de la condensación homogénea o heterogénea del $\mathrm{SO}_{2}$ atmosférico (generado en la combustión de combustibles fósiles), se depositan mediante movimientos brownianos y pueden suponer un porcentaje elevado del azufre que se encuentra depositado en las piedras expuestas.

- Las partículas medianas (entre 0,3 y $2 \mu \mathrm{m}$ ) y las grandes (entre 2 y $35 \mu \mathrm{m}$ aproximadamente), pueden ser: de naturaleza orgánica (que suponen cerca del

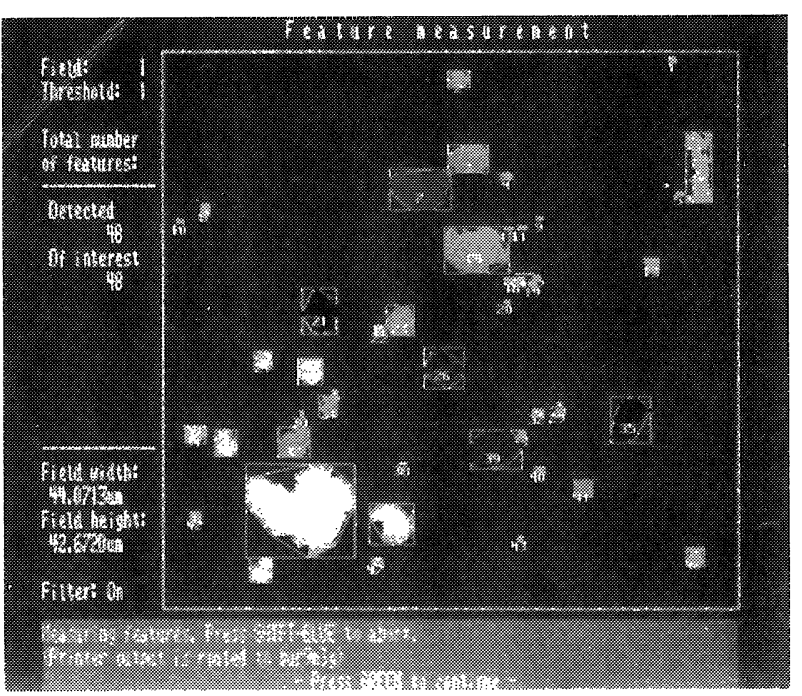

Figura 3.- Imagen de FEATURESCAN de un grupode partículas medianas (0,3-2 $\mu \mathrm{m})$. El programa permite, partiendo de una imagen "en vivo" del SEM, caracterizar de modo rápido y fiable las partículas, tanto desde el punto de vista morfológico como químico.

Figure 3.- FEATURESCAN image of a group of medium particles (0.3-2 $\mu \mathrm{m})$. The programme allows from a SEM "life" image fast and accurate both morphologic and chemical characterisation of particles.
The data obtained from both studies -stone exposure and particle collecting- give information about particle nature and their role in stone decay.

Collected particles have been analysed by SEM + EDX, including a programme for their automated characterisation (Featurescan) (Figure3). Table V shows the most significant results. In this table particles are classified by size and chemical composition.

Results show that:

Small particles (between 0.05 and $0.3 \mu \mathrm{m}$ ) form aggregates in which sulphur is the main element (sometimes almost the only one) accompanied by potassium and/or calcium (Figure 4). Those particles probably come from homogeneous or heterogeneous condensation of atmospheric $\mathrm{SO}_{2}$ (generated by fossil fuels combustion). They deposit as sulphates throughout brownian movements and can represent a higher percentage of the sulphur deposited on the stones.

Medium (0.3-2 $\mu \mathrm{m})$ and coarse (2-35 $\mu \mathrm{m})$ particles can be: particles of organic nature (around 43\%); powder or soil erosion (mirar) particles (28\%);

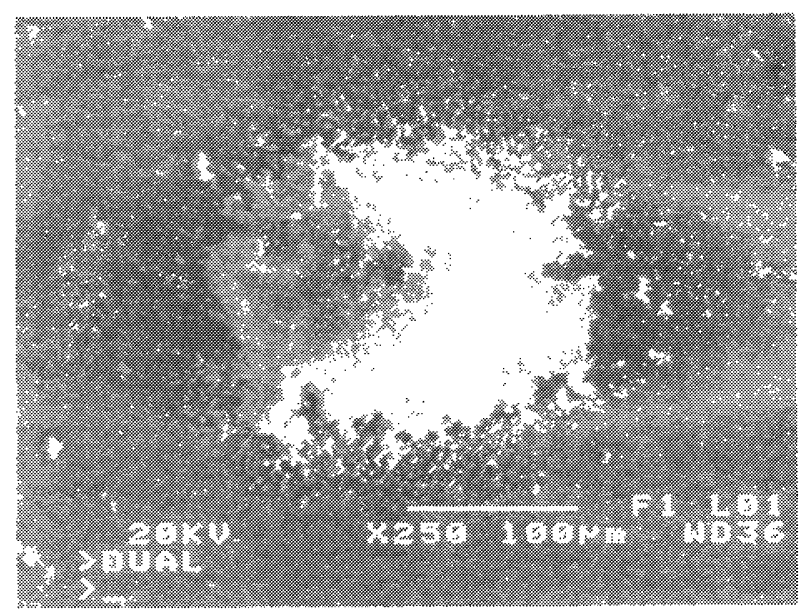

Figura 4.- Imagen de SEM de partículas pequeñas $(0,05-0,3 \mu \mathrm{m})$. En la imagen no se observan partículas aisladas, sino agregados de partículas que se han formado por condensación, locual hace imposible caracterizarlas de manera individualizada.

Figure 4.- SEM image of small particles 80.05-0.3 $\mu \mathrm{m})$. No isolated particles can be observed in this image but particle aggregates of condensation origin, therefore a individualised characterisation is impossible. 
TABLA V (TABLE V)

Clasificación y porcentajes de las partículas recogidas en la fachada de Coronería (Catedral de Burgos) (Percentage and classification of particles collected in the Coroneria factade (Burgos Cathedral))

\begin{tabular}{|c|c|c|c|}
\hline $\begin{array}{l}\text { Grupos de partículas } \\
\text { clasificadas } \\
\text { (Particle classification) }\end{array}$ & $\begin{array}{l}\text { Pequeñas } \\
\text { (Small) }\end{array}$ & $\begin{array}{l}\text { Medianas } \\
\text { (Medium) }\end{array}$ & $\begin{array}{c}\text { Grandes } \\
\text { (Large) }\end{array}$ \\
\hline $\begin{array}{c}\text { Ricas en azufre (S-rich) } \\
\text { Yeso (Gypsum) } \\
\text { S-Na } \\
\text { S-K } \\
\text { Cenizas volantes } \\
\text { (Fly ash) }\end{array}$ & $\begin{array}{l}\quad \approx 100 \\
\text { Son agregados formados } \\
\text { casi exclusivamente por } \\
\mathrm{S} \text {, con algo de } \mathrm{K} \text { y } \mathrm{Ca} \text {. } \\
\text { (The composition of the } \\
\text { aggregates is basically } S \\
\text { with small amount of } K \\
\text { and } \mathrm{Ca} \text {. }\end{array}$ & 11,6 & $\begin{array}{l}3,57 \\
2,7 \\
0,48 \\
0,39\end{array}$ \\
\hline Orgánicas (Organic) & & 42,92 & 42,95 \\
\hline $\begin{array}{l}\text { De erosión (Erosion) } \\
\text { Calcita (Calcite) } \\
\text { Cuarzo (Quartz) } \\
\text { Silicatos (Silicates) } \\
\text { Ricas en Ca (Ca-rich) } \\
\end{array}$ & & $\begin{array}{c}29,04 \\
11,94 \\
1,00 \\
3,89 \\
12,22 \\
\end{array}$ & $\begin{array}{l}27,03 \\
14,38 \\
2,61 \\
1,93 \\
8,11 \\
\end{array}$ \\
\hline $\begin{array}{c}\text { Ricas en cloro (Cl-rich) } \\
\mathrm{NaCl} \\
\text { Otros con } \\
\text { (Other with) } \\
\mathrm{Na}, \mathrm{K}, \mathrm{Ca}, \mathrm{S} \\
\end{array}$ & & $\begin{array}{l}8,83 \\
6,66 \\
2,17\end{array}$ & $\begin{array}{l}11,68 \\
9,07 \\
2,61\end{array}$ \\
\hline $\begin{array}{c}\text { Si y Al (Si and Al) } \\
\text { Combustión + silicatos } \\
\text { (fuel } \\
\text { combustion + silicates) }\end{array}$ & & 2,83 & 5,21 \\
\hline $\begin{array}{c}\text { Ricas en hierro (Fe-rich) } \\
\mathrm{Fe} \\
\mathrm{Fe}-\mathrm{Ni} \\
\mathrm{Cr}-\mathrm{Fe} \\
\end{array}$ & & $\begin{array}{l}<1,50 \\
<1,50\end{array}$ & $\begin{array}{l}1,54 \\
1,16 \\
0,10 \\
0,29\end{array}$ \\
\hline Ca y $P(C a$ and $P)$ & & 2,5 & 6,95 \\
\hline $\begin{array}{c}\text { Otras (Other) } \\
\mathrm{Al} \\
\mathrm{Cl}-\mathrm{Zn} \\
\mathrm{Na} \\
\mathrm{K} \\
\mathrm{Ti}(\mathrm{V}) \\
\mathrm{Ca}-\mathrm{Br} \\
\mathrm{Ca}+\text { metales } \\
(\mathrm{Ca}+\text { metals) }\end{array}$ & & $\begin{array}{l}1,22 \\
0,11 \\
0,11 \\
0,72 \\
0,28\end{array}$ & $\begin{array}{l}1,06 \\
0,77 \\
0,19 \\
0,10\end{array}$ \\
\hline
\end{tabular}

Partículas clasificadas como yeso pueden ser cenizas volantes, que presentan la misma composición (alto contenido en $\mathrm{S}$ y Ca). (Gypsum and fly ash particles are difficult to classifed, due to similar composition (high $S$ and Ca content/). 
$43 \%)$; partículas de erosión (= 28\%); partículas ricas en cloro $(=10 \%$ fundamentalmente $\mathrm{NaCl})$; partículas ricas en azufre $(=11 \%$ de las medianas $\mathrm{y}=4 \%$ de las grandes); partículas de silicio y aluminio; ricas en hierro; partículas de calcio y/o fósforo; y un grupo de partículas minoritarias variadas: ricas en aluminio, sodio, potasio, titanio... Entre las muestras recogidas con el captador apenas se ha detectado la presencia de cenizas volantes porosas ricas en azufre. Este tipo de partículas aparece con frecuencia sobre las muestras de piedras expuestas al ambiente. Esto se debe a que, por su gran tamaño, no son recogidas por el captador.

Al observar la morfología con SEM y analizar el quimismo con EDX, en superficie de las muestras de piedra expuestas, se detecta, sobre todos los soportes (incluido el granito), yeso como principal producto de neoformación. Aparece en cantidades muy variables y en algunas ocasiones con su característica morfología en rosetas.

$\mathrm{El}$ azufre que lo ha generado procede tanto del $\mathrm{SO}_{2}$ gaseoso, que reacciona con la piedra, como del $\mathrm{SO}_{2}$ que se deposita condensado a modo de pequeñas partículas. También puede encontrarse yeso de erosión, ya que con el captador se han detectado partículas grandes ricas en $\mathrm{S}$ posiblemente de este origen.

En cuanto al ennegrecimiento de la piedra, se ha determinado a partir de las medidas de luminosidad $\mathrm{L}^{*}$, realizadas con un colorímetro Minolta. Este parámetro no cromático, que varía entre 0 (negro) y 100 (blanco), es un indicador de la reflectancia de la superficie del material y puede utilizarse como medida del ennegrecimiento ("soiling") de los materiales debido al depósito de partículas. No hay un método concreto, pero existen expresiones matemáticas que relacionan la superficie de material cubierta por partículas con el cambio de reflectancia de la misma (22).

También existen otras expresiones basadas en la medida de reflectancia, consideradas como funciones de daño, utilizadas por diversos autores (32 y 33), que indican la velocidad o coeficiente de ennegrecemiento, y que relacionan el cambio de reflectancia con la concentración de partículas en suspensión en la atmósfera y el tiempo de exposición. Así, está comprobado que el ennegrecimiento o cambio en la reflectancia es directamente proporcional a la raiz cuadrada de dicha concentración y al tiempo de exposición: chlorine rich particles (10\%; mainly $\mathrm{NaCl}$ ); sulphur rich particles $(11 \%$ in the medium fraction and $4 \%$ in the coarse fraction); aluminosilicate particles; iron rich particles; calcium and phosphor rich particles and a small group of different particles: aluminium, sodium, potasium, titanium... rich particles.

Among the particles collected by the sampler, porous sulphur rich fly ashes are rarely detected. Particles of this kind can be observed, however, on stone samples exposed outdoors. These fly ashes are too big to be collected by the sampler.

When observing and analysing exposed stone samples under SEM-EDX, gypsum is always detected as the main decay product (even the granite ones). Gypsum amounts are variable and sometimes presents its "roseta" characteristic morphology.

The sulphur that generates this gypsum comes both from gaseous $\mathrm{SO}_{2}$ that reacts with stones and from $\mathrm{SO}_{2}$ deposited condensed as small particles. Also "soil erosion " can be the source of some of the gypsum (particles of this type have been collected by the sampler).

Stone soiling can be calculated by surface reflectance variations. In this case, the non chromatic parameter $L^{*}$ has been determined from measurements by a Minolta colorimeter. L*(lightness), which ranges from 0 (black) to 100 (white), it is an indicator of the material reflectance and can be used as an assessment of soiling. There is not a specific method, but there are some mathematical expressions that relate the coverage of material surface by particles to the change of reflectance (22).

Moreover, there are other expressions based on reflectance measurements, considered as damage functions and used by different authors $(32,33)$ that show velocity or coefficient of soiling. These functions relate reflectance changes to the TSP concentration and the exposure time. Then, it is accepted that soiling or reflectance change is "directly" proportional to this concentration and the square root of the time of exposure: donde: $\mathrm{Y}=$ reflectancia en un momento dado, $\mathrm{L}_{\mathrm{x}}(\%)$; $\mathrm{B}=$ pendiente de la curva, que en este trabajo se

$$
\mathrm{Y}=\mathrm{A}+(\mathrm{TSP})^{1 / 2} \mathrm{tB}
$$

where: $Y=$ measured percent reflectance, $L_{X}(\%)$; $B=$ slope of linear refelectance, that in this paper 
considerará como un coeficiente de ennegrecimiento (CE); TSP = media de la concentración de partículas en suspensión totales $\left(\mu \mathrm{g} / \mathrm{m}^{3}\right), t$ (tiempo de exposición) y $\mathrm{A}=$ reflectancia inicial, $\mathrm{L}_{0}(\%)$.

En la tabla VI, es donde se indica la variación de $\mathrm{L}^{*}$ en 12 meses y el RS (recubrimiento superficial por partículas). El RS se ha estimado mediante la fórmula desarrollada por Lanting (22): will be considered as a coefficient of soiling (CE), $T S P=$ average TSP concentration $\left(g / \mu \mathrm{m}^{3}\right) ; t=$ exposure time; and $A=$ initial reflectance, $L_{0}(\%)$.

Table VI shows the variation of $L^{*}$ in 12 months and $R S$ (superficial covering by particles). RS has been estimated using the formula developed by Lanting (22).

$$
\mathrm{R} / \mathrm{Ro}=(1-\mathrm{AC})+\mathrm{Rp} / \mathrm{Ro} \times \mathrm{AC}
$$

donde:

where:

$$
\mathrm{AC}=(\mathrm{Ro}-\mathrm{R}) /(\mathrm{Ro} / \mathrm{Rp})
$$

en la cual: $\mathrm{R}=$ reflectancia de la superficie sucia (\%);

Ro = reflectancia inicial de la superficie;

$\mathrm{Rp}=$ reflectancia de las partículas oscuras depositadas (\%), que se considera del $30 \% ; \mathrm{AC}=$ superficie cubierta por las partículas. A partir de aquí puede obtenerse el recubrimiento superficial efectivo (Effective area coverage) $\mathrm{EAC}=100 \% \times \mathrm{AC}$, que en este trabajo se denomina RS.

Esta expresión (recubrimiento superficial) indica el porcentaje de superficie cubierta por partículas que absorben luz (partícula negras, compuestas fundamentalmente por carbono elemental). Lanting (22). afirma que valores superiores al $5 \%$ dan lugar a serias quejas del público. being $R=$ reflectance of the soiled surface (\%); $R o=$ initial reflectance of the surface (\%); $R p=$ reflectance of the settled particles (\%), considered as $30 \% ; A C=$ fraction of area covered by particles. From this formula the effective area coverage can be obtained: $E A C=100 \% \times A C$, that in this paper are named $R S$.

The surface coating expresses the percentage of surface coated by light absorbing particles (black particles, mainly formed by elemental carbon); values above $5 \%$ provoque serious social contest. Lanting (22).

\section{TABLA VI (TABLE VI)}

Variaciones de $L^{*}$. Incremento, porcentaje y recubrimiento superficial por partículas (RAC). La última columna indica si los cambios son estadísticamente significativos $(a=0,05)$

$(L *$ variation. Increase, percentage and surface coating by particles (RS). The last column shows if

\begin{tabular}{|c|c|c|c|c|c|c|c|}
\hline & & $\begin{array}{c}L^{*} \text { inicial } \\
\text { (IL* initial) }\end{array}$ & $\begin{array}{l}L^{*} \text { final } \\
\left(L^{*} \text { finall }\right.\end{array}$ & $\Delta \mathrm{L}^{*}$ & $\begin{array}{c}\text { Variación L } \\
\text { (L Variation) } \\
(\%)\end{array}$ & $\begin{array}{l}\text { RAC } \\
\text { (\%) }\end{array}$ & $\begin{array}{l}\text { Significa- } \\
\text { tivo } \\
\text { (significa- } \\
\text { tive) }\end{array}$ \\
\hline LASPRA & $\begin{array}{l}\text { Protegida } \\
\text { (She/tered) }\end{array}$ & 86,16 & 74,20 & $-11,96$ & $-13,88$ & 21 & si (yes) \\
\hline \multirow[t]{2}{*}{ HONTORIA } & $\begin{array}{l}\text { Protegida } \\
\text { (Sheltered) }\end{array}$ & 90,23 & 76,00 & $-13,24$ & $-14,67$ & 22 & si (yes) \\
\hline & $\begin{array}{l}\text { Expuesta } \\
\text { (Exposed) }\end{array}$ & 90,43 & 74,52 & $-15,97$ & $-17,65$ & 26 & si (yes) \\
\hline \multirow[t]{2}{*}{$\begin{array}{c}\text { ROSA } \\
\text { PORRIÑO }\end{array}$} & $\begin{array}{l}\text { Protegida } \\
\text { (Sheltered) }\end{array}$ & 59,05 & 57,57 & $-1,48$ & $-2,50$ & 5 & no \\
\hline & $\begin{array}{l}\text { Expuesta } \\
\text { (Exposed) }\end{array}$ & 61,41 & 60,50 & $-0,83$ & $-1,35$ & 3 & no \\
\hline
\end{tabular}
changes are stadistically significant at $\alpha=0.05$ ) 
Partiendo de estos datos se observa que en las muestras de dolomía de Laspra y caliza de Hontoria, situadas en la Catedral de Burgos, existe una disminución en $\mathrm{L}^{*}$ que es directamente proporcional a la raíz cuadrada del tiempo (Figura 5).
Samples of Laspra dolomite and Hontoria limestone placed at Burgos cathedral show a decrease in $L^{*}$ values that are proportional to the square root of the exposure time (Figure 5).
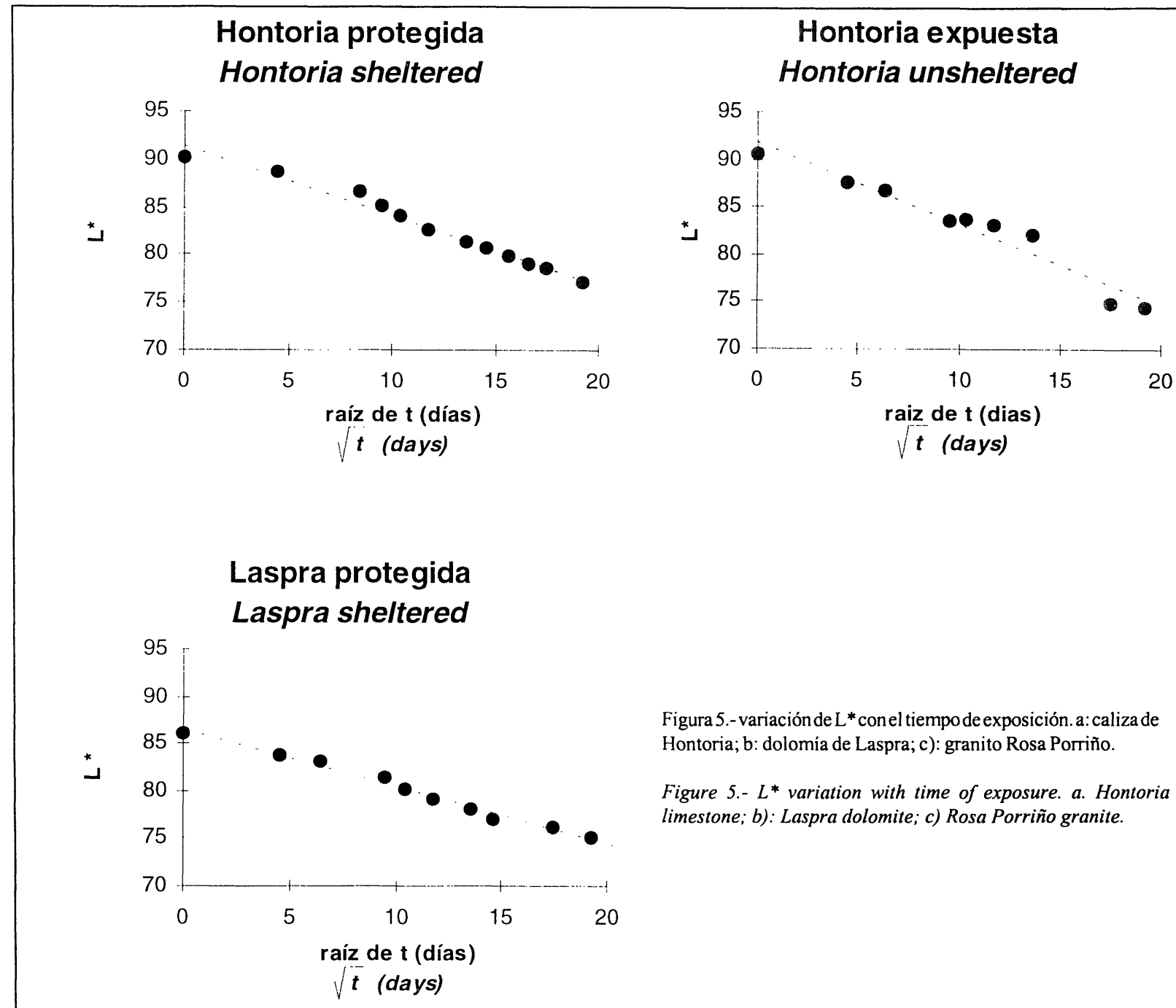

Figura 5.-variación de $L^{*}$ con el tiempode exposición. a: caliza de Hontoria; b: dolomia de Laspra; c): granito Rosa Porriño.

Figure 5.- $L^{*}$ variation with time of exposure. a. Hontoria limestone; $b$ ): Laspra dolomite; c) Rosa Porriño granite.

Rosa Porriño protegido

Rosa Porriño sheltered

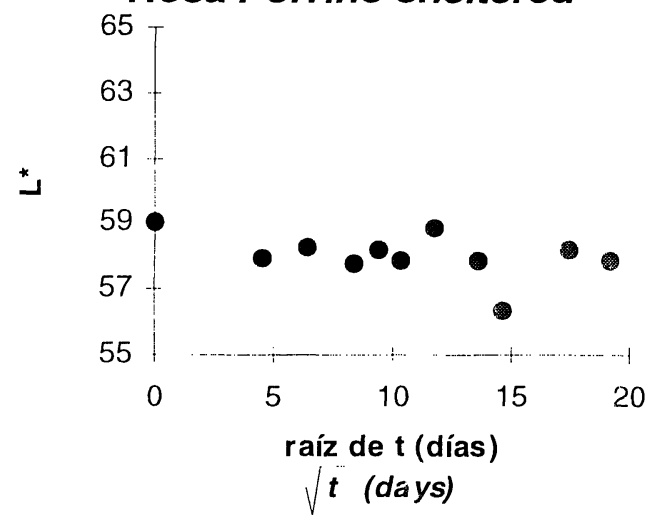

Rosa Porriño expuesto Rosa Porriño unsheltered

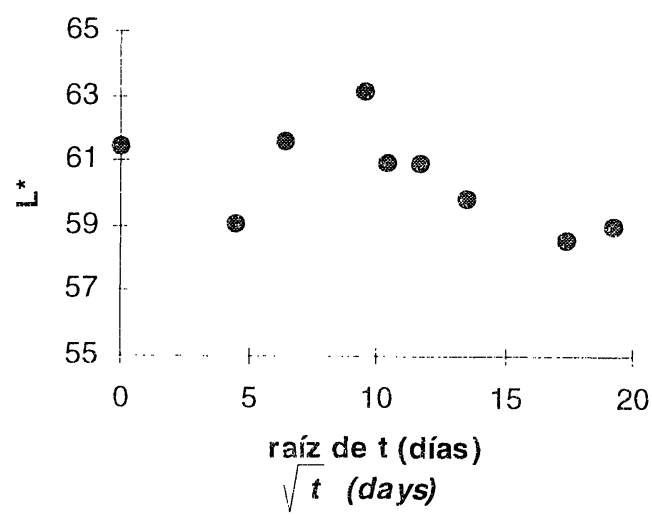


En el granito protegido de la lluvia no hay una variación significativa de este parámetro $\left(\Delta L^{*}\right)$. Sin embargo, parece que la variación de brillo (reflectancia determinada mediante brillometría) disminuye también linealmente con el tiempo, como puede verse en la figura 6.
There is not a significant $\Delta L^{*}$ variation in Rosa Porriño granite. However, it seems that gloss variation (reflectance determined by glossmeter) decreases "linealy" with time as figure 6 shows.

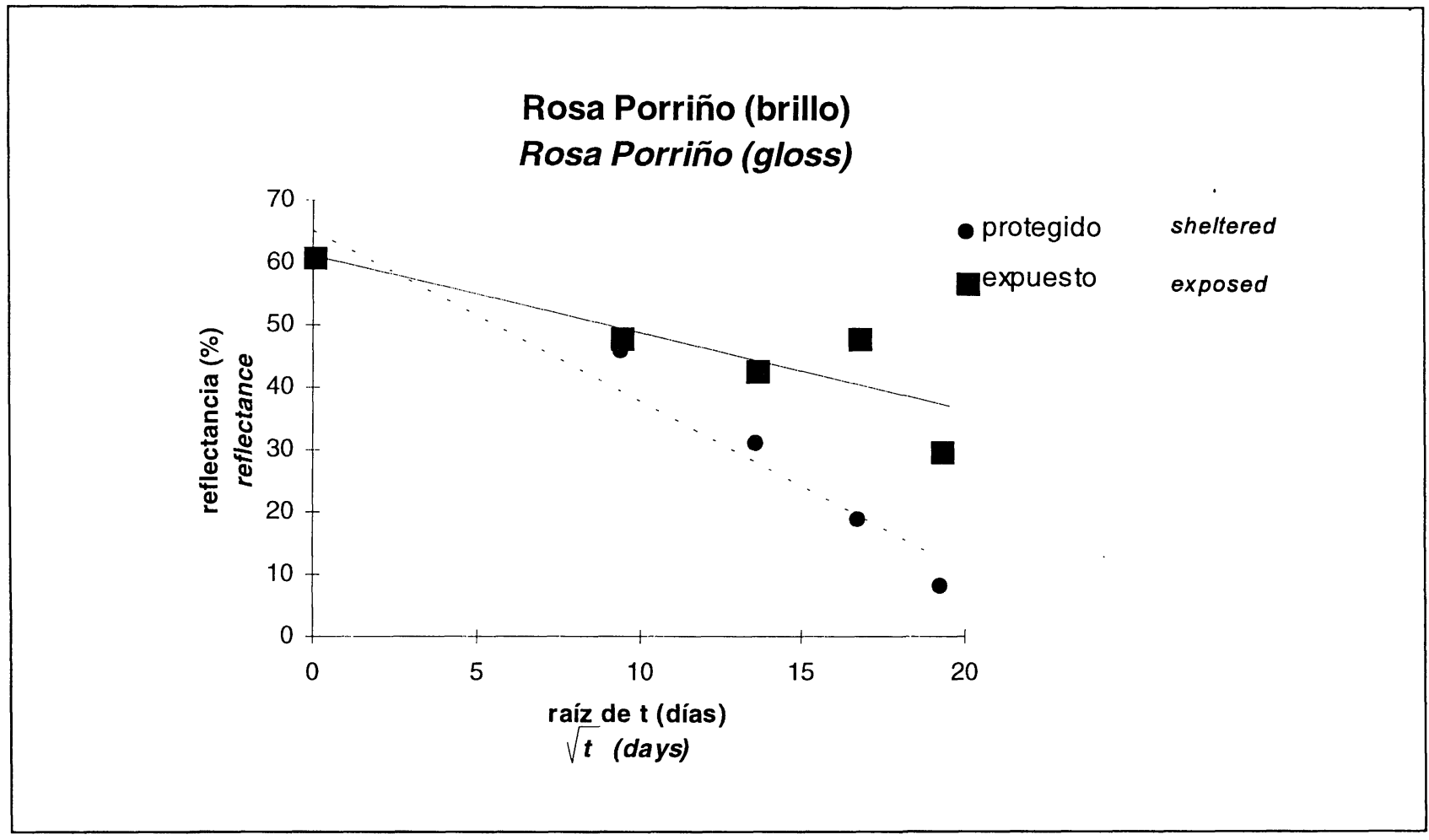

Figura 6.- variación del brillo (reflectancia) con el tiempo en muestras de granito Rosa Porriño expuestas y protegidas de la lluvia.

Figure 6.- Gloss variation (reflectance) with time of exposure of Rosa Porriño granite samples sheltered and unsheltered from rainfall.

Parece constatarse que la cantidad de partículas depositadas depende de la porosidad y rugosidad superficial, ya que en los materiales carbonatados (más porosos) el depósito de partículas es más elevado que en el granito (menos poroso); y como el ennegrecimiento parece, en general, ser igual o más acusado en Hontoria que en Laspra, que es más porosa, pero cuya rugosidad superficial es menor.

\section{ENSAYOS DE LABORATORIO. INTERPRETACIÓN DE LA ACCIÓN DE LOS AGENTES CONTAMINANTES}

Con el fin de comparar los datos obtenidos con la exposición a la intemperie con aquellos obtenidos mediante ensayos de envejecimiento simulado, se han llevado a cabo una serie de ensayos de laboratorio. Se han seleccionado algunos relacionados con la acción de los contaminantes (exposición a atmósferas
These facts corroborate that the amount of deposited particles depends on porosity and surface roughness. Particle deposition is higher in the studied carbonate materials (higher porosity) than in the granite (lower porosity). Soiling seems to be equal or higher in Hontoria than in Laspra. Laspra porosity is higher than Hontoria's, but Laspra surface roughness is lower than that of Hontoria.

\section{LABORATORY TESTS. INTERPRETATION OF THE ACTION OF POLLUTANT AGENTS}

Some laboratory tests have been carried out to compare data obtained in outside exposure tests to those obtained in simulated tests. Selected tests are related to pollutant agent action (laboratory exposure to $\mathrm{SO}_{2}$ polluted atmospheres) and to 
contaminadas de $\mathrm{SO}_{2}$ ) y con la acción de las sales solubles (cristalización de sulfato de sodio).

\subsection{Ensayos de exposición a atmósferas contaminadas de $\mathrm{SO}_{2}$}

Se han llevado a cabo en probetas de $5 \times 3 \times 1 \mathrm{~cm}$ de Laspra y Hontoria (34). Se han comparado sus resultados con los obtenidos mediante la misma metodología por otros autores en la caliza de Portland (35). Se ha simulado en una cámara climática de deposición seca de $\mathrm{SO}_{2}$ sobre superficies de piedra mojada (ciclos de humedad-sequedad) y secas. El mojado se consigue por goteo durante 8 horas seguidas por 16 horas de secado. La concentración de $\mathrm{SO}_{2}$ en la cámara fue de $2,5 \mathrm{ppm}$, que por las características de la misma equivalen a $20 \mu \mathrm{g} / \mathrm{m}^{3}$ en la atmósfera. La dolomía de Laspra sufre un importante deterioro cuando su superficie está mojada (34). En la piedra de Hontoria no se observado ninguna variación en su aspecto.

A partir de análisis cromatográficos (realizados sobre una disolución del polvo de la probeta molida en agua desionizada y del agua de escorrentía) se ha estimado la cantidad de sulfato depositado sobre las rocas (Tabla VII), tanto sobre superficies secas como mojadas. Este depósito es sensiblemente mayor sobre superficies mojadas ( 34 y 35 ). Sobre superficies secas, la deposición de $\mathrm{SO}_{2}$ puede relacionarse con la superficie específica de los materiales (Tabla I).

Sobre las superficies mojadas el producto de reacción neoformado en la dolomía de Laspra es sulfato de calcio dihidratado mientras que en la caliza de Hontoria es, principalmente, sulfito de calcio hemihidratado, aunque también se ha detectado sulfato (análisis con espectroscopía de infrarrojos).

Laspra es una micrita con un radio de acceso de poros muy pequeño, una alta superficie específica y una gran capacidad para retener agua. Esto se traduce en una soluble salt action (sodium sulphate crystallisation).

\subsection{Exposure to $\mathrm{SO}_{2}$ polluted atmosphere tests}

They have been carried out on Laspra and Hontoria specimens $(5 \times 3 \times 1 \mathrm{~cm})(34)$. Results have been compared to those obtained with the same methodology on Portland limestone (35). Dry deposition of $\mathrm{SO}_{2}$ on stone surfaces both wet (wetting-drying cycles) and dry. The wetting is attained by dripping throug 8 hours, followed by 16 hours of drying. Chamber $\mathrm{SO}_{2}$ concentration was 2.5 ppm that, in this particular case, is equivalent to an atmospheric concentration of $20 \mu \mathrm{g} /$ $m^{3}$. Decay is significant in Laspra dolomite when its surface is wet (34). No visual aspect variation is observed on Hontoria limestone.

The amount of deposited sulphate on stones has been estimated from data of ion chromatography analysis (Table VII), both on dry and wet surfaces. This deposit is considerably higher on wet surfaces $(34,35)$. Dry deposition of $\mathrm{SO}_{2}$ on dry surfaces can be related to stone specific surface area (Table I).

The new formed reaction product is calcium sulphate dihydrate on wet Laspra surfaces (more soluble magnesium sulphate is leached from the stone surface) whereas on wet Hontoria surfaces the main product is calcium sulphite hemihydrate, although some sulphate is also detected (Fourier transform infrared spectroscopy analysis).

Laspra is a micrite with very small pore opening radii, high specific surface area and high capacity to keep water. All this leads to a higher reactive surface that

TABLA VII (TABLE VII)

Cantidad estimada de sulfato depositado (mg)

(Estimate amount of deposited sulphate (mg))

\begin{tabular}{|l|c|c|c|}
\hline & LASPRA & HONTORIA & PORTLAND $^{(1)}$ \\
\hline Superficie seca (Dry surface) & 31,5 & 0,9 & 9,8 \\
\hline Superficie mojada (Wet surface) & 185 & 128 & 89 \\
\hline
\end{tabular}

1) Los datos de Portland han sido tomados de Lewry et al., 1992(34).

1) (Dates from Lewy et al., 1992/34)) 
mayor superfice reactiva que puede facilitar la oxidación de $\mathrm{SO}_{2}$. Por otra parte, la dolomía de Laspra contiene una cantidad importante de impurezas y óxidos de hierro que pueden favorecer las reacciones de oxidación (34 y 36).

\subsection{Ensayos de cristalización de sulfato de sodio.}

Se ha seleccionado el sulfato de sodio, porque es una de las sales más dañinas para los materiales rocosos debido a sus cambios de volumen al variar sus distintos estados de hidratación (decahidratado -mirabilita- o anhidro -tenardita-) y porque los sulfatos son los productos de reacción más comunes derivados de la contaminación atmosférica.

Se han llevado a cabo sobre todos los materiales carbonatados. Se realizaron 21 ciclos, las etapas de cada uno han sido: inmersión en una solución al $14 \%$ de sulfato de sodio decahidratado, secado a $60{ }^{\circ} \mathrm{C}$ y enfriamiento a $20^{\circ} \mathrm{C}$ y $65 \%$ de $\mathrm{HR}$.

El material menos durable es la dolomía de Laspra, que se desmorona en el ciclo ocho. El material más durable es la caliza de Hontoria (Figura 7) con una pérdida de material, tras los 21 ciclos, del 2,1\%. Las calizas de Portland y Combe-Down sufren, al final de los ciclos, una pérdida de peso del 5,5 y 8,8\%, respectivamente. Una vez más, la durabilidad relativa de las rocas puede relacionarse con su porosidad y su superficie específica. can make $\mathrm{SO}_{2}$ oxidation easer. Moreover, Laspra dolomite contains a high amount of impurities and iron oxides that may favour oxidation reactions $(34,36)$.

\subsection{Tests of sodium sulphate crystallisation}

Sodium sulphate has been selected because it is one of the most harmful salts to stones. The damage is induced by the volume changes with the hydration states (decahydrated -mirabilite- and anhydrous thenardite-). It can be also noticed that sulphates are the most common reaction products from atmospheric pollution.

Tests have been carried out on all the carbonate materials. 21 cycles have been performed. Each cycle consisted on the following stages: immersion in a $14 \%$ aqueous solution of sodium sulphate decahydrate, drying at $60^{\circ} \mathrm{C}$ and cooling at $20^{\circ} \mathrm{C}$ and $65 \% \mathrm{RH}$.

Laspra dolomite is the less durable material. It breaks down after 8 cycles. Hontoria limestone is the most durable material (Figure 7). Hontoria material loss, after 21 cycles is around $2.1 \%$. Portland and Combe Down weight loss is around $5.5 \%$ and $8.8 \%$ respectively. Once again, stone relative durability can be related to porosity and specific surface area.

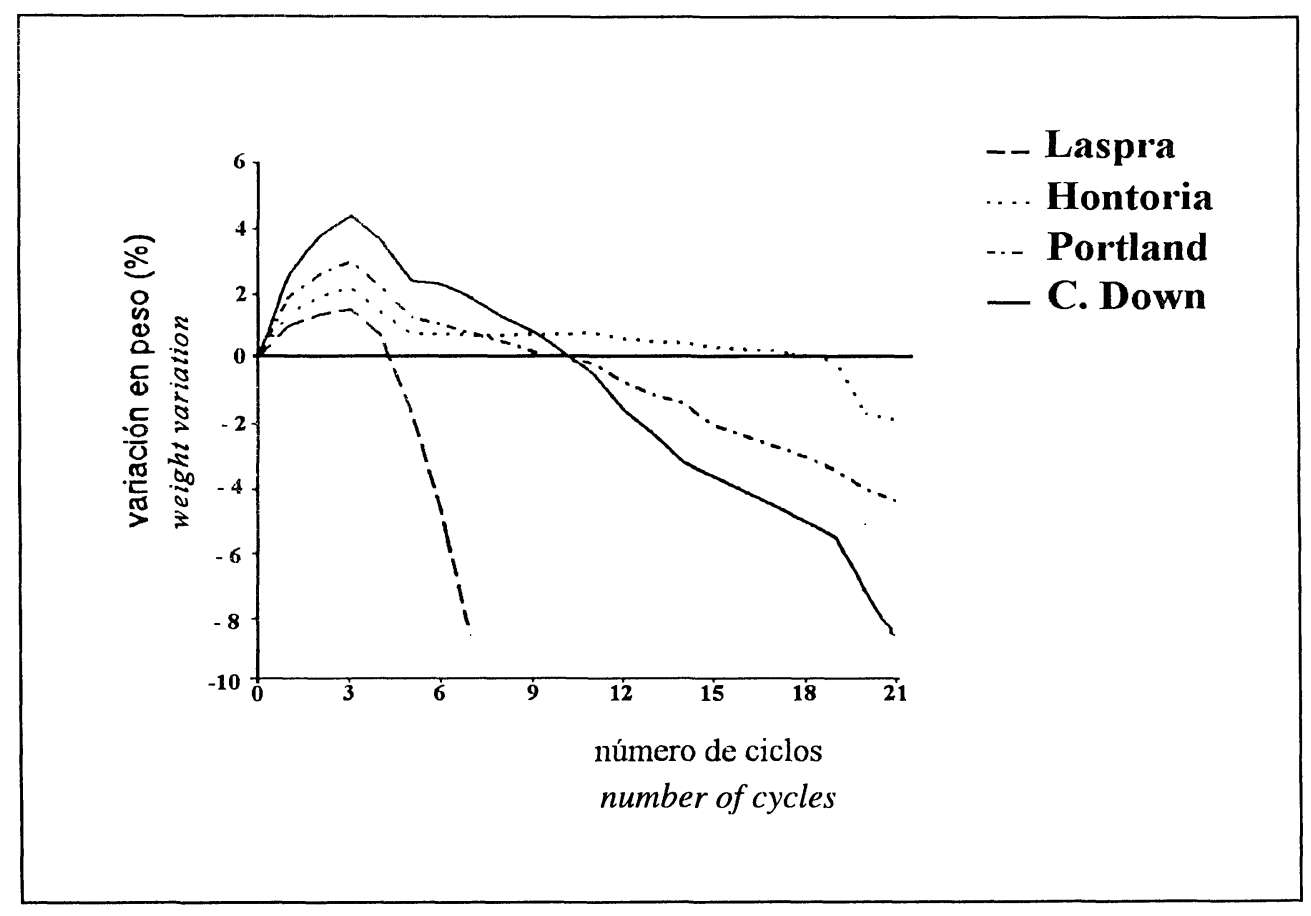

Figura 7.- Variación del peso a lo largo de los ciclos de cristalización de sales.

Figure 7.- Weight variation during salt crystallisation cycles. 


\section{CONCLUSIONES}

El deterioro de las rocas es el resultado de la combinación de una serie de agentes ambientales y de las características de las rocas. Las rocas carbonatadas son muy reactivas al agua y a la deposición seca de $\mathrm{SO}_{2}$.

En ambientes urbanos, el yeso es el principal producto de neoformación en rocas carbonatadas tanto calizas como dolomías. En la superficie de las rocas se encuentra yeso, principalmente y en menor proporción otras sales (nitratos, cloruros, etc.) de neoformación tras sólo unos meses de exposición incluso cuando el material no es carbonatado, sino silicatado. Esto corrobora la agresividad del ambiente en cuanto a la formación de productos nocivos para la conservación de la piedra.

Las propiedades físicas de las rocas carbonatadas, relacionadas con la transferencia de humedad determinan, de manera importante, su respuesta al ambiente. La porosidad abierta y la superficie específica condicionan dicha transferencia.

La porosidad abierta es importante por cuanto los espacios vacíos favorecen la entrada de agua en la roca, y con ello la disolución del carbonato. El grado de humedad superficial se ha revelado como un factor que puede multiplicar por 10 el depósito de $\mathrm{SO}_{2}$ y la velocidad de oxidación.

La distribución porosimétrica y la superficie específica influyen en la toma de humedad del aire y la retención de agua. Rocas con altas superficies específicas podran tener un alto contenido en humedad, lo que, a su vez, favorecerá la deposición seca. Esto puede tener su importancia en la oxidación del $\mathrm{SO}_{2}$ y en el depósito de $\mathrm{NO}_{2}$ que es menos soluble en agua que el $\mathrm{SO}_{2}$ ó el $\mathrm{HCl}$. Todo esto condicionará, en gran medida, la naturaleza de los productos neoformados, por ejemplo, que el producto de reacción sea sulfito o sulfato.

La deposición seca da lugar al depósito sobre la superficie de sales delicuescentes. Estas sales, por lavado o por sus características delicuescentes, pueden disolverse y migrar al interior de la roca. Si permanecen en estado líquido, harán aumentar el depósito seco debido al incremento de humedad que producen.

La disolución de los materiales carbonatados por efecto de la lluvia ácida (deposición humeda) sólo supone del 3 al 8\% del material disuelto. El resto es debido a procesos de deposición seca de $\mathrm{SO}_{2}(10-20 \%)$ y de disolución por agua natural, siendo, este último, el más importante (hasta 90\%).

\section{CONCLUSIONS}

Stone decay is the result of a combination of a series of environmental agents and stone characteristics. Carbonate stones are very reactive to unpolluted water and dry deposition of $\mathrm{SO}_{2}$.

In urban environments, gypsum is the main new formed product on carbonate stones (both limestones and dolomites). Gypsum, mainly, and, in less proportion, other salts such us nitrates or chlorides are found on stone surfaces after only a few months of outside exposure, even when the material is not carbonate but siliceous. This confirms the environment aggressiveness as far as the formation of harmful products for stone conservation.

Physical properties of stone related to moisture transfer determine to a great extent the stone response to the environment. Open porosity and specific surface area influence moisture transfer.

Open porosity is important because voids favour the access of water into stone and then carbonate dissolution. The degree of surface moisture (humidity) is a factor that can increase by 10 dry deposition of $\mathrm{SO}_{2}$ and oxidation rate.

Pore distribution and specific surface area influence the uptake of air moisture and water retention. Stones with high specific surface area can have a high moisture content which will favour dry deposition. This can be important in $\mathrm{SO}_{2}$ oxidation and NOx deposition that is less soluble in water than $\mathrm{SO}_{2}$ or $\mathrm{HCl}$. All of this will determine to a great extent the nature of the new formed products, for instance the formation of sulphate or sulphite.

Dry deposition will lead to a surface accumulation of deliquescent salts. These salts can migrate to the interior of the stone by washing and their deliquescent characteristics. In a liquid state these salts will increase the moisture in stone and therefore dry deposition.

Acid rain (wet deposition) only counts for about 3-8\% of carbonate material loss by dissolution. The rest (to $100 \%)$ correspond to dissolution by dry deposition of $\mathrm{SO}_{2}(10-20 \%)$ and natural solution, being this last process the most important in carbonate dissolution (until 90\%). 
El depósito de material particulado está condicionado por la porosidad y rugosidad de la superficie rocosa. El fenómeno de ennegrecimiento (soiling) tiene una clara relación con el depósito de partículas y se produce de forma apreciable desde los primeros meses de exposición del material pétreo a un ambiente urbano.

Las partículas sólidas de contaminación más perjudiciales para los materiales rocosos, especialmente si son de naturaleza carbonatada, son las partículas menores de 0,3 micras por su altísimo contenido en $\mathrm{S}$ y elevada superficie específica.

No obstante, y entre las de tamaños mayores, las ricas en $\mathrm{S}$ y las metálicas, juegan un papel importante porque, a pesar de ser menos numerosas, pueden funcionar como núcleos de cristalización de yeso y como catalizadores de la formación de yeso, respectivamente.

Finalmente, frente a la acción del sulfato de sodio en ensayos de cristalización de sales, el comportamiento de los materiales está claramente relacionado con su porosidad y sistema poroso. Así rocas muy porosas y con poros pequeños sufren mayores daños que rocas menos porosas y con poros de mayor tamaño. Esto ha sido corroborado tanto por los ensayos in situ como por las pruebas de laboratorio.

\section{AGRADECIMIENTOS}

Los autores agradecen el soporte económico a las siguientes instituciones: CICYT (proyecto CC95SEC0501), FICYT (proyecto PB-REC96-08) y a la Fundación Caja de Madrid. También a Matthew Murray, investigador del Building Research Lab de Garston (U.K.), así como a Araceli Rojo, de la Universidad de Oviedo, por facilitarnos los datos del granito Rosa Porriño incluidos en las tablas I y II.
Particulate matter deposition is controlled by porosity and surface roughness. The soiling process is related to particle deposition and it is perceptible only after some time of material exposure to an urban environment.

Particles smaller than 0.3 microns are the most harmful ones to stones (specially carbonate stones) due to the high $S$ content of these particles and their high specific surface area.

However, bigger $S$ rich or metallic particles can play an important role (even if they are less abundant) because they can act as nuclei of gypsum crystallisation or catalysts in the formation of gypsum, respectively.

Finally, the behaviour of materials against the sodium sulphate tests is clearly related to their porosity and pore network. In this way, stones with very high porosity and small pores are much more likely to salt decay than less porous stones and with bigger pore. This has been corroborated by in-situ and laboratory tests.

\section{ACKNOWLEDGEMENTS}

The authors wish to acknowledge the financial support of this research to CICYT (project CC95-SEC0501), FICYT (project PB-REC96-98) and "la Fundación Caja de Madrid". Also to Mr Matthew Murray, senior scientific officer from Building Research Establishment (UK), for his collaboration in the tests carried out in the UK and Ms Araceli Rojo, researcher of the "Universidad de Oviedo (Spain)" for the data of Rosa Porriño granite included in tables I and II.

\section{BIBLIOGRAFÍA}

(1) SNECK, T.: (1981). “RILEM y durability”. Matériaux et Constructions, Bull. RILEM, 83, pp. 379-390.

(2) ESBERT, R.M.; ORDAZ, J.; ALONSO, F.J. Y MONTOTO,M.: (1997). "Manual de diagnosis y tratamiento de materiales pétreos y cerámicos". Colegio de Áparejadores y Arquitectos Técnicos de Barcelona. $139 \mathrm{pp}$.

(3) ESBERT, R.M. Y MONTOTO, M.: (1995). "La petrofísica y su aplicación a los estudios de las patologías en piedra". En Patología: Conservación y Restauración de edificios- Tomo I, Colegio Oficial de Arquitectos de Madrid, pp211-230.

(4) TORRACA, G.: (1982). "Porous building materials: Materials science for architectural conservation". Roma, $141 \mathrm{p}$.

(5) GROSSI, C.M. Y MURRAY, M.: (en prensa). "Characteristics of carbonate building stones that influence the dry deposition of acid gases". Construction and Building Materials.

(6) AMOROSO, G.G. Y FASSINA, V.: (1983). "Stone Decay and Conservation". Material Science Monographs, 11. Elsevier Ed. Amsterdam, $453 \mathrm{p}$. 
(7) BERNARDIE, A.; CAMMUFFO, D.; DEL MONTE; M. y SABBIONI, G.: (1985). "Microclimate and weathering of an Historical Building: The Ducal Palace in Urbino". The Science of Total Environment, 46, pp. 246-260.

(8) PRESS, F. Y SIEVER, R.: (1988). “Weathering: The descomposition of rocks". En "Earth”, Cap. 5, pp. 101-124.

(9) ROSVALL, J.: (1988). “Air pollution and conservation”. Durability of Building Materials, Vol. 5, n.3-4 pp. 209-237.

(10) STAMBOLOW, T. y VAN ASPEREN DE BOER, J.R.J.: (1976). "The deterioration and conservation of porous building materials. A review of the literature". ICCROM, Roma, $86 \mathrm{p}$.

(11) WINKLER, E.M.: (1987). "Weathering and weathering rates of natural stone". Environmental Geology and Water Sciences, Vol. 9 , n.2, pp. 85-92.

(12) ESBERT, R.M.: (1997). “Degradación-Conservación del Patrimonio construido en piedra: Incidencia de la contaminación". En -Contaminación e Ingeniería Ambiental- Publicaciones FICYT, Vol. 5, pp. 37-51.

(13) WITTEMBURG, C. y DANNECKER, W.: (1994). "Salt enrichment in building stones by deposition of sulphur and nitrogen containing species from urban atmospheres". The Conservation of the Stone in the Mediterranean Basin, Venecia, pp. 179-183.

(14) JOHANSSON, L.G.; LINQVIST, O. y MANGIO, R.E.: (1988). "Corrosion of calcareous stones in humid air containing $\mathrm{SO}_{2}$ and $\mathrm{NO}_{2}$. Durability of Building Materials,Vol. 5, pp. 439-449.

(15) SPEDDING, D.J. (1969). Sulphur dioxide uptake by limestone, Atmospheric Environment, Vol. 3, pp. 683-685.

(10) GÖTÜRK, H.M.; VOLKAN, M. y KAHVECI, S.: (1992). "Sulfation mechanism of travertines: effect of $\mathrm{SO}_{2}$ concentration, relative humidity and temperature". Conservation of Stone and Other Materials, RILEM, Paris, Vol. 1, pp. 83-90.

(17) LEWRY, A.J; ASIEDU-DOMPREH, J.; BIGLAND, D.J. y BUTLIN, R.N.: (1994). x"The effect of humidity on the dry deposition of sulphur dioxide onto calcareous stones". Construction and Building Materials, Vol. 8, N. 2, 97-100.

(18) SPIKER, E.C.; COMER, V.J.; HOSKER, R.P. y SHERWOOD, S.I.: (1992). "Dry deposition of $\mathrm{SO}_{2}$ on limestone and marble: role of humidity". $7^{\text {the }}$ International Congress on Deterioration and Conservation of Stone, Lisboa, 397-406.

(19) VOS, B.H.: (1988). "Fundamentals of heat and moisture transfer". The Deterioration and Conservation of Stone. Studies and Documents on the Cultural Heritage. UNESCO, pp. 67-88.

(20) LIPFERT, F.W.: (1989). "Atmospheric damage to calcareous stones: comparison and reconciliation of recent experimental findings". Atmospheric Environment, Vol. 23, No 2, pp. 415-429.

(21) WEBB, A.H., BAWDEN, R.J., BUSBY, A.K. y HOPKINS, J.N.: (1992). "Studies on the effects of air pollution on limestone degradation on Great Britain". Atmospheric Environment, Vol. 26B, No 2, pp. 165-181.

(2) LANTING, R.W.: (1986). “Black smoke and soiling”. Aerosols, Lewis Publishers, Michigan, pp. 923-932.

(23) Rodríguez-Navarro, C. y Sebastian, E. (1996): "Role of particulate matter from vehicle exhaust on porous building stone (limestone) sulfation". The Sci. Total Environ. 187, pp. 79-91.

(24) GROSSI, C.M. y ESBERT, R.M.: (1994). "Las sales solubles en el deterioro de rocas monumentales". Materiales de Construcción, pp. 15-30.

(25) GROSSI, C.M.; ESBERT, R.M.; SUÁREZ DEL RÍO, L.M.; MONTOTO, M. y LAURENZI-TABASSO, M.: (1996). “Acoustic emission monitoring to study sodium sulphate crystallization in monumental porous carbonate stones". Studies in Conservation, 42, pp.115-125.

(20) BUTLIN, R.N.; COOTE, A.T.; DEVENISH, M.; HUGHES, C.M.; IRWIN, J.G.; LLOYD, G.O.; MASSEY, S.W. y YATES, T.J.S.: (1992). "A four-year study of stone decey in different pollution climates in the United Kingdom". $7^{\text {th }}$ International Congress on Deterioation and Conservation of Stone, Lisbon, pp. 345-353.

(27) ARNOLD, A. Y ZEHNDER, K.: (1989). "Salt weathering on monuments". The Conservation of Monuments in the Mediterranean Basin, Bari, pp. 31-58.

(28) AUGER, F.: (1989). "World limestone decay under marine spray conditions". The Conservation of Monuments in the Mediterranean Basin, Bari, pp. 65-69. 
(29) SMITH, B.J. Y MCGREEVY, J.P: (1988). "Contour scaling of a sandstone by salts weathering under simulated hot desert conditions". Earth Surface Processes and Landforms, Vol. 13, pp. 697-705.

(30) GROSSI, C.M.; MURRAY, M. Y BUTLIN, R.N.: (1995). "Response of porous building stones to acid deposition". Water, Soil and Air Pollution, Vol. 85, pp. 2713-2718.

(31) ESBERT, R.M.; DÍAZ-PACHE, F.; ALONSO, F.J.; ORDAZ, J. and GROSSI, C.: (1996). "Solid particles of atmospheric pollution found on the Hontoria limestone of Burgos cathedral (Spain)". $8^{\text {th }}$ International Congress on Deterioration and Conservation of Stone, Berlin, pp. 393-399.

(32) HAYNIE, F.H.: (1986). "Theorical model of soiling of surfaces by airborne particles". Aerosols, Lewis publishers, USA, pp. 951 959.

(33) YOCOM, J.E. AND KAWECKI, J.M.: (1986). "Overview of soiling and materials damage from aerosols". Aerosols, Lewis publishers, USA, pp. 913-922.

(34) GROSSI, C.M.; ESBERT, R.M.; LEWRY, A.J. y BUTLIN, R.N.: (1994). "Weathering of building carbonate rocks under $\mathrm{SO}_{2}$ polluted atmospheres". $7^{\text {th }}$ International IAEG Congress, Balkema, Rotterdam, pp. 3573-3582.

(35) LEWRY, A.J; BUTLIN, D.J. and BUTLIN, R.N.: (1992). "A chamber study of the effects of sulphur dioxide on calcareous stones". $7^{\text {th }}$ International Congress on Deterioation and Conservation of Stone, Lisbon, pp. 641-650.

(36) ESBERT, R.M. y MARCOS, R.M.: (1983). "Las piedras de la Catedral de Oviedo y su deterioración". Colegio Oficial de Aparejadores y Arquitectos Técnicos de Asturias, $147 \mathrm{pp}$.

\section{publicación del IETCC/CSIC}

\section{RESTAURACIÓN Y CONSERVACIÓN DE MONUMENTOS}

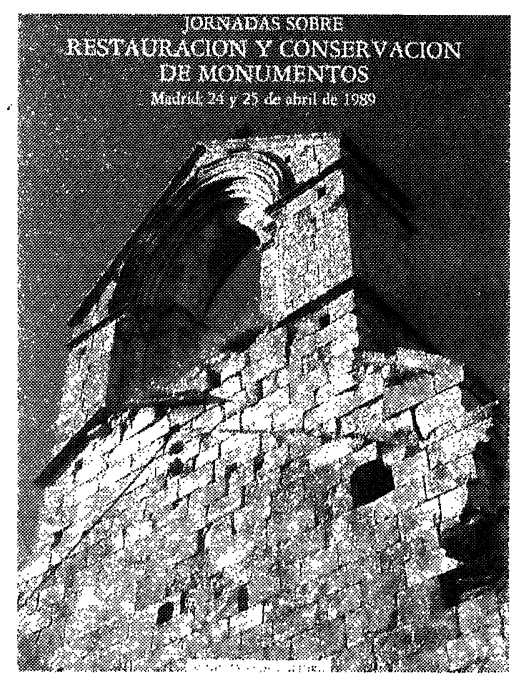

Esta publicación recoge las ponencias e intervenciones presentadas en las Jornadas sobre Restauración y Conservación de Monumentos, celebradas en el Instituto Eduardo Torroja en colaboración con el Instituto de Conservación y Restauración de Bienes Culturales, y cuyo interés y vigencia hacen de esta obra un valioso instru. mento de consulta para los profesionales interesados en la temática de la conservación y restauración del Patrimonio artístico y cultural.

Un volumen de $19,5 \times 21 \mathrm{~cm}$, 204 págs., con numerosas fotografias en color, figuras de linea, gráficos, tablas, etc. Madrid, 1991.

Existencia limitada de ejempiares.

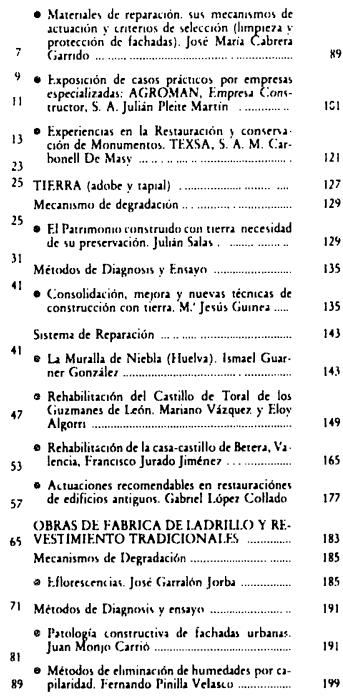

- Prologo Antono Mis-ciundal L.tatary - El Partimonio Cultural como métudo centi-
fico. Antonio Mis-Guindal Lafargat LA PIEDRA. (Porradilla) - Mecanismos de altercacion en materiales pe - Deterioro de materales pertroor por microor. Métodos de diagnosis - Aplicación de los ensyos finco mecinico

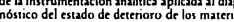

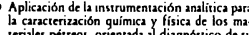
estado oe detertioro. Moists Frias Roijs - Essudio del estado de deterioro de los mate.
ristes
Maria

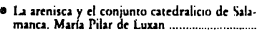
- Deterioro de lor materiales artificiales en la
Caredral de Toledo. M. Teress Blanco Varela

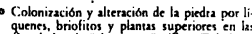

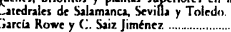

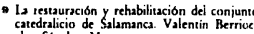
Morenes te Reparcibn

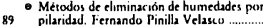

